\title{
SURGICAL EXCISION OF EYEBALL- ARE ALL CASES UNAVOIDABLE?
}

Seena A. $R^{1}$

${ }^{1}$ Associate Professor, Department of Pathology, Government Medical College, Thiruvananthapuram.

\section{ABSTRACT}

\section{BACKGROUND}

Surgical removal of eyeball is not very uncommon. It is done for different indications like tumours, inflammations, injury, etc. Some of these excisions were inevitable, but many are having modifiable courses. Such cases should be identified and the necessary measures taken to prevent the unnecessary eye loss.

This study verifies the cases of surgical removal of eyes and find out how many of them are really inevitable.

\section{MATERIALS AND METHODS}

The study type is descriptive. Evisceration, enucleation and exenteration cases admitted in a tertiary eye care centre for five years were included. The history and pathological findings were carefully studied and the nature of the indications assessed.

\section{RESULTS}

Out of 65 cases studied, 23 were malignant tumours and 2 were benign tumours, one was an eye showing degenerative changes. The remaining cases were all inflammation, injury, staphyloma, etc. which were classified as avoidable.

\section{CONCLUSION}

Among the 65 cases studied, in 33 cases excision could have been avoided if proper care was provided at the right time and six cases were due to injury which were preventable. Thus, 39 cases can be considered as avoidable. Hence, the study suggests spreading awareness regarding the care of eye.

\section{KEYWORDS}

Enucleation, Evisceration, Exenteration, Tumours, Panophthalmitis, Endophthalmitis, Blind Eye, Phthisis Bulbi.

HOW TO CITE THIS ARTICLE: Seena AR. Surgical excision of eyeball- are all cases unavoidable? J. Evolution Med. Dent. Sci. 2017;6(69):4958-4960, DOI: 10.14260/Jemds/2017/1075

\section{BACKGROUND}

The incidence of blindness had been reduced significantly after the launch of various programmes by the Government of India and their successful implementation by our network of health personnel. These were the National Programme for Control of Blindness (NPCB), ${ }^{1}$ the National Trachoma Control Programme $^{2}$ and the National Eye Health Education Programme (NEHEP). ${ }^{3}$ The NPCB, launched in 1976, mainly addresses blindness due to cataract and the cure of the same. The NEHEP was launched in 1991. It addresses blindness due to diabetic retinopathy, glaucoma and vision impairments which are not properly treated and also blindness due to the age related changes in the eye. The National Trachoma Control Programme started in 1963. In addition to these, The School Eye Health Programme was introduced to conduct screening for refractive errors and other vision defects among the school children. All these programmes were successfully implemented and proved to be effective in controlling blindness to a large extent and proved to be great achievements of our health care system over blindness. However, surgical removal of one or both eyes is still being done in significant numbers every year.

Financial or Other, Competing Interest: None.

Submission 16-07-2017, Peer Review 15-08-2017,

Acceptance 21-08-2017, Published 28-08-2017.

Corresponding Author:

Seena A. $R$,

Manusmrithi, Tc 68/2352,

NKRA 12, Manacaud P.O,

Thiruvananthapuram-695009, Kerala.

E-mail:seenaar@yahoo.com

DOI: $10.14260 /$ jemds $/ 2017 / 1075$
This includes many reasons like tumours, infections, inflammations and trauma. Many of these patients are children and young adults. In a study conducted in Nigeria, majority of eyeball excisions are done for inflammatory causes. ${ }^{4}$ In some other studies, majority of excisions were done in cases of trauma. Malignant tumours are mostly idiopathic and are absolute indications for excision. ${ }^{5}$ Similarly, eyeball that had undergone degenerative changes and become painful blind eye cannot be saved. However, the inflammatory and traumatic cases were not absolute indications like tumours. The inflammations were easily curable if they were treated in the early stages. Similarly, use of protective measures could have prevented trauma of the eyeball. Such cases were classified as avoidable.

The study was conducted in a tertiary eye care centre, to see how many of the cases of eyeball removal in our setup were inevitable and how many were avoidable. It was quite disturbing to see that most were avoidable causes, which were worsened by inadequate care given in initial stages and was rendered unavoidable. This calls for more attention and effort from health workers to prevent the loss of an eye and the associated mental trauma that occurred because of negligence.

\section{Objectives}

\section{The study objectives are}

1. To study the indications of surgical removal of the eyeball in a period of five years.

2. To classify these cases as avoidable and inevitable.

3. To find out how many of the cases were preventable or avoidable with proper protective measures and better care in early stages. 


\section{MATERIALS AND METHODS}

The study type is descriptive. All cases of eyeball excision that were done at the centre over a period of 5 years from 2012 to 2017 were included in the study.

The history of the cases was studied in detail and the microscopic appearances were scrutinised.

Depending on these observations, the indications were classified as inevitable and avoidable and the results tabulated.

The percentage of eyeball excisions that were avoidable was calculated.

Centre of the study: Regional Institute of Ophthalmology, Thiruvananthapuram.

\section{RESULTS}

A total number of sixty-five cases of eyeball excision were obtained during the period of study from 2012 to 2017. Eleven cases were in 2012, twelve cases in 2013, fourteen cases in 2014, fifteen cases in 2015 and thirteen in 2016. Among the patients, sixteen were children below the age of 12 years and eight patients were under the age group between 13 years and 45 years. Thus, children and young adults constituted twenty-four cases, that is thirty seven percent. This fact highlights the relevance of this study.

Among the sixty-five cases, twenty-three were malignant tumours, retinoblastoma being the leading cause accounting for thirteen cases, melanoma came second that is six cases and squamous cell carcinoma following with four cases. Fourteen cases were under the age of ten, thirteen with retinoblastoma and one case for bilateral squamous cell carcinoma. This case of bilateral squamous carcinoma was very unusual, since squamous cell carcinoma is seldom seen in this age group. A detailed study was conducted to find out whether there were any predisposing factors or if the child was having the tumour as a component of any syndromes. But no such connections were identified. Of the rest of the neoplasms, among adults six were melanoma and three were squamous cell carcinoma.

\begin{tabular}{|c|c|c|c|}
\hline Indications & $\begin{array}{c}\text { Number } \\
\text { of Cases }\end{array}$ & $\mathbf{\%}$ & $\begin{array}{c}\text { Avoidable/ } \\
\text { Inevitable }\end{array}$ \\
\hline Retinoblastoma & 13 & 20 & Inevitable \\
\hline Melanoma & 6 & 9.5 & Inevitable \\
\hline Squamous carcinoma & 4 & 6.5 & Inevitable \\
\hline Benign tumours & 2 & 3 & Inevitable \\
\hline Atrophy & 1 & 1.5 & Inevitable \\
\hline Nonspecific inflammation & 20 & 31 & Avoidable \\
\hline Staphyloma & 8 & 12 & Avoidable \\
\hline Perforating injury & 6 & 9 & Avoidable \\
\hline Suppuration & 3 & 4.5 & Avoidable \\
\hline Granulomatous inflammation & 2 & 3 & Avoidable \\
\hline \multicolumn{4}{|c|}{ Table 1. Classification of Cases } \\
\hline
\end{tabular}

Benign tumours like neurofibroma and capillary haemangioma were also seen. In normal course, these do not need eyeball excision as excision of the tumour will be curative. However, the benign tumours in this study were causing symptoms which necessitated surgical removal of eye. The neurofibroma was plexiform neurofibroma, which was a part of Von Recklinghausen's disease. It grew to large size causing significant disfigurement and had to be removed along with the eye. The capillary haemangioma on the other hand was small in size. But being a vascular tumour it developed bleeding that was intractable, thus making excision inevitable.

The leading non-neoplastic cause for eyeball removal was inflammation, accounting for 20 cases. Of these five were corneal abrasions to start with, treated by some other systems of medicine leading to secondary infection and extensive ulceration. Three cases were due to foreign body in the eye, which were neglected. They remained in the eye causing chronic irritation and got infected. The rest of the inflammations all started as infections of the eye like conjunctivitis and keratitis. These were locally treated with some herbals, which failed to control the infections. This gradually led to dissemination of the infection. By the time they reached the hospital, the eyes had become atrophic and painful. In short, all cases of inflammations were preventable. This was followed by Staphyloma with haemorrhage, which includes eight cases. It is a protrusion of uveal tract through weakened sclera. In our study, the sclera was weakened because of previous infections. Hence, they were considered avoidable. Rest of the cases included six cases of injury and three cases of suppuration. Two cases of granulomatous inflammation were there; one of which was due to tuberculous infection and the other was associated with fungal mass in the eyeball. Atrophic eyeball due to longstanding inflammation in an old diabetic accounted for the remaining one case.

\section{DISCUSSION}

Enucleation, exenteration and evisceration are the common methods of eyeball removal. Evisceration is the least radical procedure among these. It means scraping out the contents of the eyeball, leaving the sclera intact. As the sclera is intact, the shape of the eyeball is preserved to some extent. The procedure is done in cases of penetrating trauma, painful bleeding eye and endophthalmitis. Enucleation removes the globe after dividing the conjunctiva and extrinsic muscles. This is typically done in cases like intraocular tumours, phthisis bulbi and sympathetic ophthalmia. Exenteration, the most mutilating of the three, removes the entire content of the orbit, the globe, extrinsic muscles and the periosteum. It is done in malignant tumours of the eyeball, orbit, paranasal sinuses or eyelid with invasion of surrounding structures. These surgeries result in psychological stress, because of partial loss of vision and the disfigurement that is caused. Hence, it would be better if these destructive surgeries are avoided as far as possible.

With this aim, the cases were classified as avoidable cases and unavoidable ones. The neoplastic lesions are mostly idiopathic and their course is not predictable or modifiable. Furthermore, to prevent spread of the tumour and its recurrence, malignant tumours like squamous cell carcinoma and melanoma are to be removed along with wide margins, which demand eyeball excision. Hence, they were classified under unavoidable indications. The commonest intraocular tumour, retinoblastoma affects children below the age of 5 yrs. Most cases involve only eyeball, but it can spread to the optic nerve and through it may reach the brain. Though it occurs due to the $\mathrm{Rb}$ gene mutation, it cannot be predicted and by no means it can be prevented. Hence, it qualifies as unavoidable indication. Inflammations, on the other hand, most cases started as mild infections, corneal abrasions or 
mild injuries. Five of them were corneal abrasions, which were treated locally according to some indigenous medicine. The abrasions which were easily treatable, became infected and transformed into ulcers. By the time they reached the hospital, the eyes were showing panophthalmitis. Although, efforts were made to save the eyes, it was not possible since the inflammation became intractable. Some cases were initially infections, like keratitis or conjunctivitis. They were treated with some leaves or oils by quacks. The infections spread to involve the whole globe so that the eyeballs were reduced to abscesses, phthisis bulbi making surgery inevitable. These cases, if given proper treatment, at the right time would have been cured completely. Hence, they were included under avoidable causes. Indications like degenerative conditions leading to staphyloma, suppurative infections in immunocompromised like old age, diabetes, etc. were included as inevitable along with tumours.

Some of the cases were penetrating injuries that if left as such can lead to sympathetic ophthalmia and loss of vision in the uninjured eye too. These are hence absolute indications for surgery. All the same, these are preventable too. We had six cases of injuries; three of them were due to accidents at workplaces. One was due to a blast injury in an accident during fireworks in a festival, where precautions that should have been observed were grossly neglected. Two cases were children, who sustained injury while playing with sharp objects. This was reminiscent of a series of eyeball injuries that occurred way back in nineties due to imitation of the fights in the popular epic that was being telecasted then. Such mishaps can be easily avoided by avoiding sharp toys. The accidents in workplace can be avoided by wearing protective glasses, as specified in the company rules. Hence, they can be included under avoidable causes.
Among the cases 33 were inflammations, infections and abrasions which ended up in excision because of improper treatment in initial stages. This constitutes 52\%. Perforating injuries were six in number, making eight percentage. Hence, the avoidable causes were estimated as $60 \%$, that is 39 cases.

\section{CONCLUSION}

The various programmes launched by the Government in the field of eye health have been successful to a large extent in preventing blindness. However, surgical excision of eyeball is still continuing to be a cause of partial loss of vision and stress. According to this study, sixty percent of these surgeries could have been avoided by better protective measures for eyes and by proper treatment of the infections and inflammations of eyes at the right time. Hence, the health care personnel should emphasise on these points and create an awareness among people regarding their eye care so that loss of eyes for preventable reasons could be avoided.

\section{REFERENCES}

[1] Verma R, Khanna P, Prinja S, et al. The national programme for control of blindness in India. AMJ 2011;4(1):1-3.

[2] Preobragenski VV, Gupta UC. The national trachoma control programme in India. Indian journal of ophthalmology 1964;12(2):68-73.

[3] Silver K, Macario E. The national eye health education program: increasing awareness of diabetic eye disease among American Indians and Alaska natives. Ethnicity \& Disease 2006;16:920-5.

[4] Koylu MT, Gokce G, Uysal Y, et al. Indications for eye removal surgeries. Saudi Med J 2015;36(10):1205-9.

[5] Lim KH. Absolute indication for the removal of an eye. Medical journal 1973;14(1):9-12. 